\title{
NONCONVEX MINIMIZATION IN GENERATING SPACE
}

\author{
VANITA BEN DHAGAT AND VIJENDRA SINGH THAKUR
}

\begin{abstract}
A nonconvex minimization theorem has been established for generating of space of quasi 2-metric family with non commuting condition, which is defined as $\alpha$-compatible.
\end{abstract}

\section{Introduction and preliminary}

The generating space of quasi 2-metric family is important because of its involvement with fuzzy and probabilistic 2-metric space and a minimization theorem [1], [2] is to obtain fixed point theorem. In this paper, we prove a non convex minimization theorem for generating space of quasi 2-metric family. Further, we prove fixed point theorem as an application of minimization theorem with non commuting condition Known as $\alpha$-compatible. Generating space of quasi 2-metric family is defined as follows:-

Let $X$ be a non empty set and $\left\{D_{\alpha}: \alpha \in(0,1]\right\}$ be family of mapping $D_{\alpha}$ from $X x X x X$ into $R^{+} .\left\{X, D_{\alpha}\right\}$ is called generating space of quasi 2-metric family if it satisfy following axioms:

(GM1) - For any two distinct points $x$ and $y$ there exists $z$ in $X$ such that $D_{\alpha}(x, y, z) \neq 0$, $\forall \alpha \in(0,1]$.

(GM2) - $D_{\alpha}(x, y, z)=0$ if at least two $x, y, z$ are equal and $\alpha \in(0,1]$.

(GM3) - $D_{\alpha}(x, y, z)=D_{\alpha}(x, z, y)=D_{\alpha}(z, y, x)$ for all $x, y, z$ in $X$ and $\alpha \in(0,1]$.

(GM4) - For any $\alpha \in(0,1]$ there exists $\alpha_{1}, \alpha_{2}, \alpha_{3} \in(0, \alpha]$ such that $\alpha_{1}+\alpha_{2}+\alpha_{3} \leq \alpha$ and so $D_{\alpha}(x, y, z) \leq D_{\alpha 1}(x, y, u)+D_{\alpha 2}(x, u, z)+D_{\alpha 3}(u, y, z)$.

(GM5) - $D_{\alpha}(x, y, z)$ is non increasing and left continuous in $\alpha$ and $\forall x, y, z$ in $X$.

Throughout this paper, we assume that $K:(0,1] \rightarrow(0, \infty)$ is a non decreasing function satisfying the condition

$$
K=\sup _{\alpha} K(\alpha) .
$$

Let $E$ and $F$ be mappings from generating space of quasi 2-metric family $\left\{X, D_{\alpha}\right\}$ into itself. The mapping $E$ and $F$ are said to be $\alpha$-compatible if

$$
\lim _{n \rightarrow \infty} D_{\alpha}\left(E F x_{n}, F E x_{n}, E E x_{n}\right)=0 \text { and } \lim _{n \rightarrow \infty} D_{\alpha}\left(E F x_{n}, F E x_{n}, F F x_{n}\right)=0
$$

Received February 5, 2007.

2000 Mathematics Subject Classification. 54H25, 47H10.

Key words and phrases. Generating space of quasi 2-metric family, $\alpha$-compatible mapping, Minimization theorem, common fixed point. 
for all $\alpha \in(0,1]$ and $x_{n}$ is a sequence in $X$ such that $\lim _{n \rightarrow \infty} E x_{n}=\lim _{n \rightarrow \infty} F x_{n}=t$ for some $t$ in $X$.

Lemma 1. Let $E$ and $F$ be $\alpha$-compatible mappings from generating space of quasi 2-metric family $\left\{X, D_{\alpha}\right\}$ into itself. Suppose that $\lim _{n \rightarrow \infty} E x_{n}=\lim _{n \rightarrow \infty} F x_{n}=t$ for some $t$ in $X$. Then

(i) $\lim _{n \rightarrow \infty} E F x_{n}=F t$ if $F$ is continuous.

(ii) $E F t=F E t$ and $E t=F t$ if $E$ and $F$ are continuous.

Proof. (i) Suppose that $\lim _{n \rightarrow \infty} E x_{n}=\lim _{n \rightarrow \infty} F x_{n}=t$ for some $t$ in $X$. Since $F$ is continuous, we have $\lim _{n \rightarrow \infty} F E x_{n}=F t$. Now by (GM4), we have

$$
D_{\alpha}\left(E F x_{n}, F t, F t\right) \leq D_{\alpha 1}\left(E F x_{n}, F t, F E x_{n}\right)+D_{\alpha 2}\left(E F x_{n}, F E x_{n}, F t\right)+D_{\alpha 3}\left(F E x_{n}, F t, F t\right)
$$

For $\alpha_{1}, \alpha_{2}, \alpha_{3} \in(0, \alpha]$ such that $\alpha_{1}+\alpha_{2}+\alpha_{3} \leq \alpha$. On taking limit $n \rightarrow \infty$ we get $\lim _{n \rightarrow \infty} E F x_{n}=F t$. Since $E$ is continuous, $\lim _{n \rightarrow \infty} E F x_{n}=E t$ and also $F$ is continuous so by (i) $\lim _{n \rightarrow \infty} E F x_{n}=F t$. Hence by uniqueness $E t=F t$. Now again $D_{\alpha}(E F t, F E t, E F t) \leq \lim _{n \rightarrow \infty} D_{\alpha}\left(E F x_{n}, F E x_{n}, E F x_{n}\right)$ for all $\alpha \in(0,1]$. Since $E$ and $F$ and by (GM2) we get $E F t=F E t$.

\section{Main result}

Theorem 1. Let $\left\{X, D_{\alpha}^{\prime}: \alpha \in(0,1]\right\}$ and $\left\{Y, D_{\alpha}^{\prime}: \alpha \in(0,1]\right\}$ be two complete generating space of quasi 2-metric family. $F: X \rightarrow Y$ be a closed and $T: X \rightarrow X$ be continuous mapping satisfying

(i) $D_{\alpha}(T x, T y, z) \leq \max \left\{D_{\alpha}(T x, y, z), D_{\alpha}(x, T y, z), D_{\alpha}(x, y, T z)\right\}$ and

(ii) $D_{\alpha}^{\prime}(f(T x), f(T y), f(z)) \leq \max \left\{D_{\alpha}^{\prime}(f(T x), f(y), f(z)), D_{\alpha}^{\prime}(f(x), f(T y), f(z))\right.$,

$$
\left.D_{\alpha}^{\prime}(f(x), f(y), f(T z))\right\}, \forall x, y, z \text { in } X \text { and } \alpha \in(0,1]
$$

(iii) $\psi: \Re \rightarrow \Re$ be a non decreasing continuous and bounded below function,

(iv) $\phi: f(X) \rightarrow \Re$ be a lower semi continuous and bounded below function,

(v) for any $p \in X$ with $\inf _{x^{\prime} \in X} \psi(\phi(f(x)))<\psi(\phi(f(p)))$ there exists $q$ with $p \neq T q$ and $\max \left[\max \left\{D_{\alpha}(T q, p, z), D_{\alpha}(q, T p, z), D_{\alpha}(q, p, T z)\right\}, c \cdot \max \left\{D_{\alpha}^{\prime}(f(T q), f(p), f(z))\right.\right.$, $\left.\left.D_{\alpha}^{\prime}(f(q), f(T p), f(z)), D_{\alpha}^{\prime}(f(q), f(p), f(T z))\right\}\right] \leq K(\alpha)[\psi(\phi(f(p)))-\psi(\phi(f(q)))]$ $\forall x, y, z$ in $X$ and $\alpha \in(0,1]$ and $c$ is any constant.

Then there exists an $x_{0}$ in $X$ such that $\inf _{x \in X} \psi(\phi(f(x)))=\psi\left(\phi\left(f\left(x_{0}\right)\right)\right)$.

Proof. Let us suppose $\inf _{x \in X} \psi(\phi(f(x)))<\psi(\phi(f(y)))$ for every $y$ in $X$ and choose $r \in X$ for which $\psi(\phi(f(r)))$ is defined, then inductively we define a sequence $\left\{r_{n}\right\} \subset X$ with $r_{1}=r$. Suppose $r_{n}$ is known and consider

$$
\begin{aligned}
W_{n}= & \left\{\left[w \in X: \max \left[\max \left\{D_{\alpha}\left(T w, r_{n}, z\right), D_{\alpha}\left(w, T r_{n}, z\right), D_{\alpha}\left(w, r_{n}, T z\right)\right\},\right.\right.\right. \\
& \left.c \cdot \max \left\{D_{\alpha}^{\prime}\left(f(T w), f\left(r_{n}\right), f(z)\right), D_{\alpha}^{\prime}\left(f(w), f\left(T r_{n}\right), f(z)\right), D_{\alpha}^{\prime}\left(f(w), f\left(r_{n}\right), f(T z)\right)\right\}\right] \\
\leq & K(\alpha)\left[\psi\left(\phi\left(f\left(r_{n}\right)\right)\right)-\psi(\phi(f(w)))\right] \quad \forall x, y, z \text { in } X \text { and } \alpha \in(0,1] .
\end{aligned}
$$


$W_{n}$ is non empty set and there exists $w \in W_{n}$ such that $r_{n} \neq T w$. We can choose $r_{n+1} \in W_{n}$ such that and $r_{n} \neq T\left(r_{n+1}\right)$ and

$$
\psi\left(\phi\left(f\left(r_{n+1}\right)\right)\right) \leq \inf _{x \in X} \psi(\phi(f(x)))+1 / 3\left[\psi\left(\phi\left(f\left(r_{n}\right)\right)\right)-\inf _{x \in X} \psi(\phi(f(x)))\right] .
$$

Clearly $\psi\left(\phi\left(f\left(r_{n+1}\right)\right)\right)$ is a non increasing lower bounded sequence, hence it is a convergent sequence.

Now we prove $\left\{r_{n}\right\}$ and $\left\{f\left(r_{n}\right)\right\}$ are Cauchy sequences:

$$
\begin{aligned}
\max \left\{D_{\alpha}\left(T r_{n}, T r_{n+1}, w\right), c . D_{\alpha}^{\prime}\left(f\left(T r_{n}\right), f\left(r_{n+1}\right), f(w)\right)\right\} \\
\leq \max \left[\max \left\{D_{\alpha}\left(T r_{n}, r_{n+1}, w\right), D_{\alpha}\left(r_{n}, T r_{n+1}, w\right), D_{\alpha}\left(r_{n}, r_{n+1}, T w\right)\right\},\right. \\
\quad c \cdot \max \left\{D_{\alpha}^{\prime}\left(f\left(T r_{n}\right), f\left(r_{n+1}\right), f(w)\right), D_{\alpha}^{\prime}\left(f\left(r_{n}\right), f\left(T r_{n+1}\right), f(w)\right),\right. \\
\left.\left.\quad D_{\alpha}^{\prime}\left(f\left(r_{n}\right), f\left(r_{n+1}\right), f(T w)\right)\right\}\right] \\
\leq K(\alpha)\left[\psi\left(\phi\left(f\left(r_{n}\right)\right)\right)-\psi\left(\phi\left(f\left(r_{n+1}\right)\right)\right)\right]
\end{aligned}
$$

$\forall n, m \in N, n<m \Rightarrow$ there exists $\alpha_{j}=\alpha_{j}(n, m) ; \sum \alpha_{j} \leq \alpha$, such that

$$
\begin{gathered}
\max \left\{\max \left\{D_{\alpha}\left(T r_{n}, r_{m}, w\right), D_{\alpha}\left(r_{n}, T r_{m} w\right), D_{\alpha}\left(r_{n}, r_{m}, T w\right)\right\}\right. \\
\quad c \cdot \max \left\{D_{\alpha}^{\prime}\left(f\left(T r_{n}\right), f\left(r_{m}\right), f(w)\right), D_{\alpha}^{\prime}\left(f\left(r_{n}\right), f\left(T r_{m}\right), f(w)\right),\right. \\
\left.\left.D_{\alpha}^{\prime}\left(f\left(r_{n}\right), f\left(r_{m}\right), f(T w)\right)\right\}\right\} \\
\leq \sum_{j=n} \max \left\{\max \left\{D_{\alpha j}\left(T r_{j}, r_{j+1}, w\right), D_{\alpha j}\left(r_{j}, T r_{j+1}, w\right), D_{\alpha j}\left(r_{j}, r_{j+1}, T w\right)\right\}\right. \\
\quad c \cdot \max \left\{D_{\alpha j}^{\prime}\left(f\left(\operatorname{Tr}_{j}\right), f\left(r_{j+1}\right), f(w)\right), D_{\alpha j}^{\prime}\left(f\left(r_{j}\right), f\left(T r_{j+1}\right), f(w)\right),\right. \\
\left.\left.D_{\alpha j}^{\prime}\left(f\left(r_{j}\right), f\left(r_{j+1}\right), f(T w)\right)\right\}\right\}
\end{gathered}
$$

Hence, $\forall n, m \in N, n<m$ and $\mu<\alpha$ :

$$
\begin{gathered}
\max \left\{\max \left\{D_{\alpha}\left(T r_{n}, r_{m}, w\right), D_{\alpha}\left(r_{n}, T r_{m}, w\right), D_{\alpha}\left(r_{n}, r_{m}, T w\right)\right\}\right. \\
c \cdot \max \left\{D_{\alpha}^{\prime}\left(f\left(T r_{n}\right), f\left(r_{m}\right), f(w)\right), D_{\alpha}^{\prime}\left(f\left(r_{n}\right), f\left(T r_{m}\right), f(w)\right),\right. \\
\left.\left.D_{\alpha}^{\prime}\left(f\left(r_{n}\right), f\left(r_{m}\right), f(T w)\right)\right\}\right\} \\
\leq K(\mu) \sum_{j=n}^{m-1}\left[\psi\left(\phi\left(f\left(r_{j}\right)\right)\right)-\psi\left(\phi\left(f\left(r_{j+1}\right)\right)\right)\right] \\
\leq K(\alpha)\left[\psi\left(\phi\left(f\left(r_{n}\right)\right)\right)-\psi\left(\phi\left(f\left(r_{m}\right)\right)\right)\right]
\end{gathered}
$$

for some $\alpha_{j}$ with $0<\alpha_{j+1}<\alpha_{k} \leq \alpha, j=n, \ldots, \ldots, m-1$.

$$
\begin{aligned}
D_{\alpha}\left(r_{n}, r_{n+1}, w\right) & \leq D_{\alpha 1}\left(r_{n}, r_{n+1}, T r_{n+1}\right)+D_{\alpha 2}\left(r_{n}, T r_{n+1}, w\right)+D_{\alpha 3}\left(T r_{n+1}, r_{n+1}, w\right) \\
& \leq D_{\alpha 1}\left(r_{n}, r_{n+1}, T r_{n+1}\right)+D_{\alpha 2}\left(r_{n}, T r_{n+1}, w\right)+D_{\alpha 3}\left(T r_{n+1}, r_{n+1}, T r_{n}\right)
\end{aligned}
$$




$$
\begin{aligned}
& +D_{\alpha 4}\left(\operatorname{Tr}_{n+1}, T r_{n}, w\right)+D_{\alpha 5}\left(T r_{n}, r_{n+1}, w\right) \\
& \text { for } \alpha_{1}+\alpha_{2}+\alpha_{3}+\alpha_{4}+\alpha_{5} \leq \alpha \\
\leq & 3\left[\max \left\{D_{\alpha}\left(T r_{n}, r_{n+1}, w\right), D_{\alpha}\left(r_{n}, T r_{n+1}, w\right), D_{\alpha}\left(r_{n}, r_{n+1}, T w\right)\right\},\right. \\
& \quad \cdot \max \left\{D_{\alpha}^{\prime}\left(f\left(\operatorname{Tr}_{r n}\right), f\left(r_{n+1}\right), f(w)\right), D_{\alpha}^{\prime}\left(f\left(r_{n}\right), f\left(T r_{n+1}\right), f(w)\right),\right. \\
& \left.\left.D_{\alpha}^{\prime}\left(f\left(r_{n}\right), f\left(r_{n+1}\right), f(T w)\right)\right\}\right] \\
\leq & 3 K(\alpha)\left[\psi\left(\phi\left(f\left(r_{n}\right)\right)\right)-\psi\left(\phi\left(f\left(r_{n+1}\right)\right)\right)\right] .
\end{aligned}
$$

Then also we get

$$
D_{\alpha}\left(r_{n}, r_{m}, w\right) \leq 3 K(\alpha)\left[\psi\left(\phi\left(f\left(r_{n}\right)\right)\right)-\psi\left(\phi\left(f\left(r_{m}\right)\right)\right)\right] \quad \text { where } n<m \text {. }
$$

In the manner we obtain

$$
D_{\alpha}^{\prime}\left(f\left(r_{n}\right), f\left(r_{n+1}\right), f(w)\right) \leq 3 K(\alpha)\left[\psi\left(\phi\left(f\left(r_{n}\right)\right)\right)-\psi\left(\phi\left(f\left(r_{m}\right)\right)\right)\right] \quad \text { where } n<m .
$$

Hence $\left\{r_{n}\right\}$ and $\left\{f\left(r_{n}\right)\right\}$ are Cauchy sequences.

Assume that $\lim _{n \rightarrow \infty} r_{n}=a$ and $\lim _{n \rightarrow \infty} f\left(r_{n}\right)=b$. Since $f$ is closed therefore $f(a)=b$

By the continuity of $\Psi$ and lower semi continuity of $\phi$ we have

$$
\psi(\phi(b)) \leq \lim _{n \rightarrow \infty} \psi\left(\phi\left(f\left(r_{n}\right)\right)\right)=\lim _{n \rightarrow \infty} \psi\left(\phi\left(f\left(r_{n+1}\right)\right)\right) .
$$

Let $\delta=\inf _{x \in X} \psi(\phi(f(x))) \in \mathfrak{R}$

$$
\psi\left(\phi\left(f\left(r_{n+1}\right)\right)\right) \leq \inf _{x \in X} \psi(\phi(f(X)))+1 / 3\left[\psi\left(\phi\left(f\left(r_{n}\right)\right)\right)-\inf _{x \in X} \psi(\phi(f(x)))\right],
$$

we have

$$
\begin{gathered}
\lim _{n \rightarrow \infty} \psi\left(\phi\left(f\left(r_{n+1}\right)\right)\right) \leq(2 / 3) \delta+1 / 3 \lim _{n \rightarrow \infty} \psi\left(\phi\left(f\left(r_{n}\right)\right)\right)=(2 / 3) \delta+1 / 3 \lim _{n \rightarrow \infty} \psi\left(\phi\left(f\left(r_{n+1}\right)\right)\right) \\
\psi(\phi(f(a)))<\psi(\phi(b)) \leq \lim _{n \rightarrow \infty} \psi\left(\phi\left(f\left(r_{n+1}\right)\right)\right) \leq \delta=\inf _{x \in X} \psi(\phi(f(x))) \leq \psi(\phi(f(a)))
\end{gathered}
$$

which is contradiction, therefore there exists $x_{0}$ in $X$ such that

$$
\inf _{x \in X} \psi(\phi(f(x)))=\psi\left(\phi\left(f\left(x_{0}\right)\right)\right) .
$$

Now we give a fixed point theorem as an application of the above theorem under non commuting condition known as $\alpha$-compatible.

Theorem 2. Let $\left\{X, D_{\alpha}: \alpha \in(0,1]\right\}$ and $\left\{Y, D_{\alpha}^{\prime}: \alpha \in(0,1]\right\}$ be two complete generating space of quasi 2-metric family. $F: X \rightarrow Y$ be a closed and $T: X \rightarrow X$ be continuous mapping satisfying

(i) $D_{\alpha}(T x, T y, z) \leq \max \left\{D_{\alpha}(T x, y, z), D_{\alpha}(x, T y, z), D_{\alpha}(x, y, T z)\right\}$ and 
(ii) $D_{\alpha}^{\prime}(f(T x), f(T y), f(z)) \leq \max \left\{D_{\alpha}^{\prime}(f(T x), f(y), f(z)), D_{\alpha}^{\prime}(f(x), f(T y), f(z))\right.$,

$$
\left.D_{\alpha}^{\prime}(f(x), f(y), f(T z))\right\},
$$

(iii) $\psi: \mathfrak{R} \rightarrow \mathfrak{R}$ be a non decreasing continuous and bounded below function,

(iv) $\phi: f(X) \rightarrow \mathfrak{R}$ be a lower semi continuous and bounded below function,

(v) $S: X \rightarrow X$ be a continuous mapping,

(vi) $S$ and $T$ are $\alpha$ compatible and

$$
\begin{gathered}
\max \left[\max \left\{D_{\alpha}(T x, T S x, z), D_{\alpha}(x, T S x, z), D_{\alpha}(x, S x, T z)\right\},\right. \\
\quad c \cdot \max \left\{D_{\alpha}^{\prime}(f(T x), f(T S x), f(z)), D_{\alpha}^{\prime}(f(x), f(T S x), f(z)),\right. \\
\left.\left.D_{\alpha}^{\prime}(f(x), f(S x), f(T z))\right\}\right] \\
\leq K(\alpha)[\psi(\phi(f(x)))-\psi(\phi(f(S x)))]
\end{gathered}
$$

$\forall x, y, z$ in $X$ and $\alpha \in(0,1]$ and $c$ is any constant. Then there exists unique common fixed point $x_{0}$ in $X$.

Proof. If $x_{0} \in X$ such that $\inf _{x \in X} \psi(\phi(f(x)))=\psi\left(\phi\left(f\left(x_{0}\right)\right)\right)$ then $x_{0}=T S x_{0}, S x_{0}=T x_{0}$ and also $x_{0}=T x_{0}$. By the hypothesis, if $x_{0} \neq T x_{0}$ or $S x_{0} \neq T x_{0}$. Then for some $\alpha \in(0,1]$

$$
\begin{aligned}
0 & <\max \left\{D_{\alpha}\left(T x_{0}, T S x_{0}, z\right), D_{\alpha}\left(x_{0}, T S x_{0}, z\right), D_{\alpha}\left(x_{0}, S x_{0}, T z\right)\right\} \\
& \leq K(\alpha)\left[\psi\left(\phi\left(f\left(x_{0}\right)\right)\right)-\psi\left(\phi\left(f\left(S x_{0}\right)\right)\right)\right] \leq 0
\end{aligned}
$$

which contradiction. Then $S x_{0}=T x_{0}$. Now by $\alpha$ compatibility of $S$ and $T$ and by the lemma we get $S x_{0}=T S x_{0}=S T x_{0}=T x_{0}$.

Also for $\alpha_{1}, \alpha_{2}, \alpha_{3} \in(0, \alpha]$ such that $\alpha_{1}+\alpha_{2}+\alpha_{3} \leq \alpha$

$$
\begin{aligned}
D_{\alpha}\left(x_{0}, T x_{0}, z\right) & \leq D_{\alpha 1}\left(x_{0}, T x_{0}, T S x_{0}\right)+D_{\alpha 2}\left(x_{0}, T S x_{0}, z\right)+D_{\alpha 3}\left(T S x_{0}, T x_{0}, z\right) \\
& \leq D_{\alpha 3}\left(T S x_{0}, T x_{0}, z\right)=0 . \quad \text { Hence } T x_{0}=S x_{0}=x_{0} .
\end{aligned}
$$

Uniqueness. Let us assume there exists another fixed point $y_{0}$ such that $S y_{0}=T y_{0}=y_{0}$ and by the Theorem 1 we have $\inf _{x \in X} \psi(\phi(f(x)))=\psi\left(\phi\left(f\left(y_{0}\right)\right)\right)$.

But $\inf _{x \in X} \psi(\phi(f(x)))=\psi\left(\phi\left(f\left(x_{0}\right)\right)\right)$ hence by uniqueness of infima we get $x_{0}=y_{0}$.

Corollary 1. Let $\left\{X, D_{\alpha}: \alpha \in(0,1]\right\}$ and $\left\{Y, D_{\alpha}^{\prime}: \alpha \in(0,1]\right\}$ be two complete generating space of quasi 2-metric family. $F: X \rightarrow Y$ be a closed, $\phi: f(X) \rightarrow \mathfrak{R}$ be a lower semi continuous and bounded below function. Let $S: X \rightarrow X$ be a mapping such that

$$
\max \left\{D_{\alpha}(S x, x, z), c . D_{\alpha}^{\prime}(f(S x), f(x), f(z))\right\} \leq K(\alpha)[\phi(x)-\phi(S x)]
$$

$\forall x, y, z$ in $X$ and $\alpha \in(0,1]$ and $c$ is any constant. Then there exists $S x_{0}=x_{0}$.

Proof. Considering $T=I$ and $\psi=I$ we get required result. 


\section{Example}

Let $X=[0,1]$ and $Y=[0, \infty], D_{\alpha}=D_{\alpha}^{\prime}=D_{1}$ defined by $D_{1}(x, y, z)=\frac{D(x, y, z)}{1+D(x, y, z)}$ and $D(x, y, z)=\max \{|x-y|,|y-z|,|z-x|\}$. The mappings are defined as follows: $T: X \rightarrow X$ as $T x=x^{2}, f: X \rightarrow X$ as $f x=x, \phi: f(X) \rightarrow R$ as $\phi(x)=1 /(1-x)$ and $\psi: R \rightarrow R, \psi(x)=x^{2} / 2$ and $K(\alpha)=3$ satisfy the all conditions of Theorem 1 . Also $S: X \rightarrow X$ is defined $S x=x^{3} / 2$, then $(S, T)$ is $\alpha$ compatible which satisfying the condition of Theorem 2 and hence 0 is a unique fixed point.

\section{Acknowledgement}

Authors thank to Madhya Pradesh Council of Science and Technology (MAPCOST) for financial support through Project M-19/2006.

\section{References}

[1] D. Downing and W. A. Kirk, A generalization of Cariti's theorem with applications in nonlinear mapping theory, Pacific J. Math. 69(1977), 339-346.

[2] J. S. Jung, Y. J. Cho and J. K. Kim, Minimization theorems for fixed point theorems in fuzzy metric space and application, Fuzzy Sets and System 61(1994), 199-270.

Department of Mathematics, Jai Narain College of Technology, Bairasia Road, Bhopal M. P., India.

E-mail: vanital_dhagat@yahoo.co.in

E-mail: vijendra@yahoo.co.in 